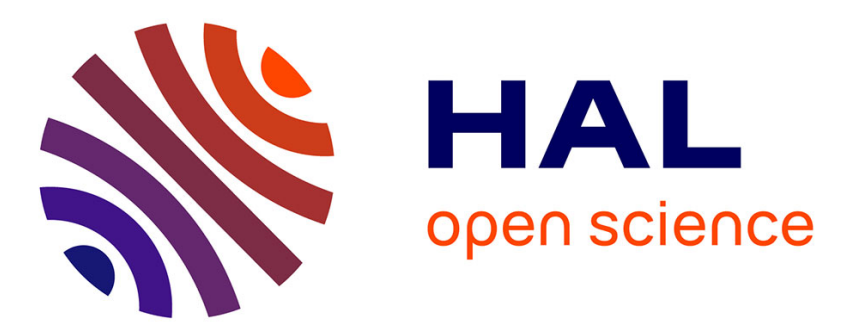

\title{
The seed-specific heat shock factor A9 regulates the depth of dormancy in Medicago truncatula seeds via ABA signaling
}

Julia Zinsmeister, Souha Berriri, Denise Puntel Basso, Benoît Ly Vu, Thi Thu

Dang, David Lalanne, Edvaldo Aparecido Amaral Silva, Olivier Leprince, Julia Buitink

\section{To cite this version:}

Julia Zinsmeister, Souha Berriri, Denise Puntel Basso, Benoît Ly Vu, Thi Thu Dang, et al.. The seedspecific heat shock factor A9 regulates the depth of dormancy in Medicago truncatula seeds via ABA signaling. Plant, Cell and Environment, 2020, Early Access, 15 p. 10.1111/pce.13853 . hal-02911644

\section{HAL Id: hal-02911644 \\ https://hal.inrae.fr/hal-02911644}

Submitted on 4 Aug 2020

HAL is a multi-disciplinary open access archive for the deposit and dissemination of scientific research documents, whether they are published or not. The documents may come from teaching and research institutions in France or abroad, or from public or private research centers.
L'archive ouverte pluridisciplinaire HAL, est destinée au dépôt et à la diffusion de documents scientifiques de niveau recherche, publiés ou non, émanant des établissements d'enseignement et de recherche français ou étrangers, des laboratoires publics ou privés. 


\title{
The seed-specific heat shock factor A9 regulates the depth of dormancy in Medicago truncatula seeds via ABA signaling
}

\author{
Julia Zinsmeister ${ }^{1}$, Souha Berriri², Denise Basso ${ }^{3}$, Benoit Ly Vu ${ }^{1}$, Thi Thu Dang ${ }^{2}$, David \\ Lalanne $^{2}$, Edvaldo AA Silva ${ }^{3}$, Olivier Leprince ${ }^{1}$, and Julia Buitink ${ }^{2}$ \\ ${ }^{1}$ AGROCAMPUS OUEST \\ ${ }^{2}$ INRAE \\ ${ }^{3}$ UNESP
}

May 14, 2020

\begin{abstract}
During the later stages of seed maturation, two key adaptive traits are acquired that contribute to seed lifespan and dispersal, longevity and dormancy. The seed-specific heat shock transcription factor A9 is an important hub gene in the transcriptional network of late seed maturation. Here we demonstrate that HSFA9 plays a role in thermotolerance rather than in ex situ seed conservation. Storage of hsfa9 seeds of Medicago truncatula and Arabidopsis had comparable lifespan at moderate storage relative humidity $(\mathrm{RH})$, whereas at high $\mathrm{RH}$, hsfa9 seeds lost their viability much faster than wild type seeds. Furthermore, we show that in $M$. truncatula, Mthsfa9 seeds acquired more dormancy during late maturation than wild type. Transient expression of MtHSFA 9 in hairy roots and transcriptome analysis of Mthsfa 9 Tnt1 insertion mutants identified a deregulation of genes involved in ABA biosynthesis, catabolism and signalling. Consistent with these results, Mthsfa 9 seeds exhibited increased ABA levels and higher sensitivity to ABA. These data suggest that in legumes, HSFA9 acts as a negative regulator of the depth of seed dormancy during seed development via the modulation of hormonal balance.
\end{abstract}

\section{Acknowledgements}

We thank the ANAN technical platform for access to their equipment.

\section{INTRODUCTION}

During development, seeds acquire a set of physiological characteristics that are essential to ensure the dispersion of the species and are therefore crucial for the establishment of seedlings in the field. These characteristics include dormancy and seed longevity, which play complementary roles in maintaining the embryo in a protected state in anticipation of favorable conditions to ensure germination (Finch-Savage \& Bassel 2015; Penfield 2017; Leprince, Pellizzaro, Berriri \& Buitink 2017). Both traits vary markedly among plant species with important consequences on plant phenology, establishment in the field, yield andex situ conservation of the genetic diversity. Dormancy is an adaptive trait that inhibits freshly matured seeds to germinate under otherwise favorable conditions or out of the appropriate season. During storage and/or dispersal, mature seeds progressively lose dormancy over time, a process known as after-ripening. Longevity is defined as the ability to survive for extended periods of time during dry storage for seed crops and in soil seed banks for wild species (reviewed in Longet al. 2015; Sano et al. 2016; Leprince et al.2016). Longevity depends on the ability of a seed to undergo complete desiccation without loss of viability. Dormancy and longevity are acquired progressively during seed maturation and are under the control of the maternal environment and mainly abscisic acid (ABA) (Graeber, Nakabayashi, Miatton, Leubner-Metzger \& Soppe 2012; Zinsmeister et al. 2016; Penfield \& MacGregor 2017). 
In legumes, the acquisition of dormancy and longevity occurs during late seed maturation concomitantly with the upregulation of heat shock proteins (HSP) and small HSPs (sHSP) (Verdier et al. 2013; Limaet al. 2017). The expression of HSP17.4 during seed maturation in Arabidopsis parallels the acquisition of dormancy and desiccation tolerance, and desiccation-intolerant mutants have decreasedHSP17.4 levels (Wehmeyer, Hernandez, Finkelstein \& Vierling 1996; Wehmeyer \& Vierling 2000). Several functional studies point to a role of sHSP in seed stress tolerance. In cabbage, the amount of HSP17.6 in dry seeds was positively correlated with germination under water stress conditions and after storage at $10 \%$ moisture and $42^{\circ} \mathrm{C}$ (Bettey \& Finch-Savage 1998). Arabidopsis and rice seeds overexpressing OsHSP18.2 , a sHSP that accumulates during late maturation, display an improved tolerance to controlled deterioration (CD) when stored at $100 \% \mathrm{RH}$ and $45^{\circ} \mathrm{C}$ and to osmotic stress during germination (Kaur et al. 2015). sHSPs are thought to act as ATP-independent chaperones that bind stress-denaturing proteins to prevent their irreversible aggregation. The ATP-dependent chaperone machinery, composed of HSP70, HSP101 and other cochaperones can then proceed to refold sHSP-associated proteins (reviewed in (Ohama, Sato, Shinozaki \& Yamaguchi-Shinozaki 2017; Jacob, Hirt \& Bendahmane 2017).

Accumulation of HSPs is under the transcriptional control of heat shock factors (HSF), a large family of transcriptional factors represented by highly conserved structural features such as a N-terminal DNA binding domain (DBD) and an oligomerization domain composed of two hydrophobic heptad repeats (HRA and HR-B) connected to DBD. Clade A of HSFs are characterized by the exclusive presence of AHA transactivator motif in their C-terminal trans-activation domain. To exert their function in stress tolerance, HSFAs are activated by ABA and function together with a set of transcription factors such as DREB2A and additional co-chaperones (Kotak, Vierling, Baumlein \& Von Koskull-Dorlng 2007; Huang, Niu, Yang \& Jinn 2016; Jacob et al. 2017; Bulgakov, Wu \& Jinn 2019). Functional diversification was established among different HSFA members (Chauhan, Khurana, Agarwal \& Khurana 2011; Jacob et al. 2017; Bulgakov et al. 2019). HSFA9 represents a unique member of the HSFA family that is specifically expressed in seeds during development and under the regulatory control of ABA INSENSITIVE 3 (ABI3) without the need of a heat shock (Kotak et al. 2007). Seed-specific overexpression of the HSFA9 from Helianthus annuum in tobacco activated the expression of various HSPs (HSP101, sHSP-CI, sHSP-CII, and plastid sHSP) and transgenic seeds exhibited increased resistance to CD (Prieto-Dapena, Castano, Almoguera \& Jordano 2006). In contrast, HaHSFA9 repression in tobacco seeds using an active repressor version HSFA9-SRDX resulted in the reduction of seed-specific sHSP proteins and a decreased tolerance against CD but did not affect desiccation tolerance (Tejedor-Cano et al. 2010), suggesting a specific role in seed longevity. Additional phenotypes observed in HaHSFA9 overexpression lines are related to the protection of the photosynthesis apparatus from dehydration and oxidative stress in tobacco seedlings (Almoguera et al. 2012) or tolerance to severe water loss (Prieto-Dapena, Castano, Almoguera \& Jordano 2008).

In Medicago truncatula, the construction of a gene regulatory network of transcription factors preferentially expressed in seeds identified a homologue of HSFA9 as the connecting node between longevity and desiccation tolerance modules (Verdier et al.2013). The objective of this work was to investigate the role of HSFA9 in the regulation of seed longevity and vigor in M. truncatula. In the dry state, seeds can survive for many years, making it difficult to assess their longevity within a reasonable time (Hay, Valdez, Lee, Sta Cruz \& Sta. Cruz 2018). To accelerate the deterioration of seeds during storage in a tractable time scale, CD or accelerating ageing tests have been proposed by increasing the $\mathrm{RH}$ at or above $85 \%$ and temperatures over 40degC during storage, conditions used to assess the role of HSFA9 in previous studies. However, there are increasing concerns from biophysics and genetics studies about the reliability of these tests as a proxy to evaluate seed longevity ex situ in conditions typically found in gene banks where the low RH and temperature allowed the seeds to enter into a solid-like state as opposed to a fluid state (reviewed in (Leprince et al. 2017; Hayet al. 2018; Ballesteros \& Walters 2019; Zinsmeister, Leprince \& Buitink 2020). Here we discovered that seed survival was only affected in hsfa9 mutants from M. truncatula and Arabidopsis when water contents in the seeds exceeded approx. $0.2 \mathrm{~g} \mathrm{H}_{2} \mathrm{O}$ g DW, showing that HSFA9 has a role in thermotolerance during wet storage, when the cytoplasm is in a fluid state. Our study also reveals an unexpected role for MtHSFA9 in the regulation of the depth of seed dormancy in M. truncatula. Further analysis of putative targets 
indicated a strong deregulation of genes involved in ABA and gibberellin (GA) metabolism and signaling, translated in an increase in ABA content and hypersensitivity to ABA in the Mthsfa9 seeds.

\section{Experimental procedures}

\section{Plant material and growth conditions}

Three independent Tnt1 insertion lines in the HSFA gene Medtr4g126070 of M. truncatula, Mthsfa9-1 (NF10440), 6 bp from ATG), Mthsfa9-2 (NF13157, 46 bp from ATG) and Mthsfa9-3 (NF12877,1333 bp from ATG) in the R108 background were obtained after screening by the Noble Foundation. Homozygous lines were screened by PCR with the primers listed in Table S1. For each allele, wild type control plants were simultaneously selected based on the absence of the Tnt1 insertion in the MtHSFA9 gene and are referred to as associated wild type. The homozygous Mthsfa9-1line was backcrossed once with the pollen of the wild type R108. Arabidopsis insertion lines in AtHSFA9 (At5g54070) were obtained from ABRC. Among the three T-DNA insertion lines ordered, homozygous lines were only identified for the Salk_062453 using primers in Table S1.

M. truncatula plants from the different genotypes were grown in batched for 10 plants trays containing a sterile mix of vermiculite at $20 \mathrm{degC} / 19 \mathrm{degC}$, with a $16 \mathrm{~h}$ light photoperiod at $200 \mu \mathrm{mol}$ photons $\mathrm{m}^{2} \mathrm{~S}^{2}{ }^{2}$. For the high temperature treatment during seed development, plants were transferred to a growth room at $26^{\circ} \mathrm{C}$ with similar light conditions when 10 flowers had appeared. Flowers were tagged and developing seeds were harvested at different time intervals until pod abscission and after final desiccation. At the abscission stage the pods were dried for 3 days at a relative humidity of $44 \%$ generated by saturated solution of $\mathrm{K}_{2} \mathrm{CO}_{3}$ at $20^{\circ} \mathrm{C}$. The seeds were manually removed from the pods and stored at $20^{\circ} \mathrm{C}$ in the dark for further afterripening or kept at $-20^{\circ} \mathrm{C}$. Arabidopsis plants were grown at $20^{\circ} \mathrm{C} / 18^{\circ} \mathrm{C}$ with a photoperiod of 16 hours. The seeds were harvested after pod abscission and then dried for three days at $44 \% \mathrm{RH}$, generated by saturated solution of $\mathrm{K}_{2} \mathrm{CO}_{3}$ at $20{ }^{\circ} \mathrm{C}$.

\section{Physiological tests}

For all tests, three replicates of 30 seeds each for M. truncatula or three replicates of 150-200 seeds each of Arabidopsis were used unless mentioned otherwise. To test for germination, M.truncatula seeds were scarified with a sandpaper and imbibed in the dark at $20^{\circ} \mathrm{C}$ (Chatelain et al. 2012). Seeds of A. thaliana were imbibed at $20^{\circ} \mathrm{C}$ and a photoperiod of 16 hours light. For both species, germinated seeds were scored at regular time intervals by counting seeds having an emerged radicle with length [?] $1 \mathrm{~mm}$. To release physiological dormancy prior to germination tests, scarified seeds of $M$. truncatula were imbibed at $20 \mathrm{deg} \mathrm{C}$ for $4 \mathrm{~h}$ in Petri dishes onto filter papers then transferred at $4 \operatorname{deg} \mathrm{C}$ for three days in the dark. For A. thaliana, seeds were imbibed for 3 days directly at $4 \mathrm{degC}$ in the dark. Water contents were assessed gravimetrically for triplicate samples of 5 seeds by determination of the fresh weight and subsequent dry weight after $2 \mathrm{~d}$ in an oven at $96 \mathrm{degC}$. Water contents are expressed on a dry weight basis. Seed weight of the genotypes was measured on five replicates of 50 seeds after oven drying at $96 \mathrm{deg} C$ for $2 \mathrm{~d}$.

For accelerated aging assays, scarified seeds of $M$. truncatula or mature seeds of Arabidopsis were placed over a saturated solution of $\mathrm{NaCl}(75 \% \mathrm{RH})$ at $35 \mathrm{degC}$ in hermetically sealed boxes and viability was evaluated by germination at regular time intervals. Longevity was expressed as P50, defined as the time (days) at which the stored seed lost $50 \%$ of viability during storage. Controlled deterioration assays were carried out by equilibration of seeds at $85 \% \mathrm{RH}$ (saturated $\mathrm{KCl}$ ) or incubation in water vapor $(100 \%)$ and $35 \mathrm{deg} \mathrm{C}$ or 40degC.

The effect of ABA inhibition on the T50, the time of imbibition needed to reach $50 \%$ of germination, was determined by imbibition of mature seeds of $M$. truncatula in a $10 \mu \mathrm{M}$ fluridone solution freshly prepared from a $1 \mathrm{mM}$ stock solution where fluridone was dissolved in ethanol and Tween 20 solution $(2 / 1 \mathrm{v} / \mathrm{v})$.

ABA sensitivity was determined by imbibition of mature, 4 months after ripened seeds on filter paper on a range of ABA concentrations (mixed isomers, Sigma, St Louis, MO, USA) at $20^{\circ} \mathrm{C}, 16 \mathrm{~h}$ light or in the dark. ABA was dissolved in methanol prior to dilution in water. Control seeds were imbibed in the $\mathrm{MeOH}$ 
concentration corresponding to the highest ABA concentration (0.05\% MeOH). Germination was scored after 14 days.

To evaluate dormancy model of the Mthsfa9 mutants is imposed by the embryo or by the surrounding tissues (testa, endosperm) or combinations of these tissues, three replicates of 30 seeds were imbibed for 6 hours at $20^{\circ} \mathrm{C}$. Using a magnifying glass, the endosperm and seed coat were carefully removed, taking care of not damaging the embryos. Scarified intact seeds and naked embryos were imbibed in water at $25^{\circ} \mathrm{C}$ in the dark. Germination (for intact seeds) or embryo growth were recorded over time.

\section{Soluble sugar quantification}

Three replicates of 30 seeds each were lyophilized and dry weight was determined gravimetrically. Sugars were extracted and analysed by HPLC on a Carbopac PA-1 column (Dionex Corp.) as described by Rosnoblet et al. (2007).

\section{ABA quantification}

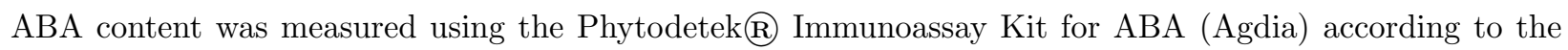
manufacturer's instructions. Extraction was performed on 3 biological replicates of 25 mature seeds 1 month after harvest according to Jiang and Zhang (2001).

\section{RNA extraction and Quantitative real time PCR}

RNA extraction was performed on 3 replicates of 30 seeds each that were ground in liquid nitrogen using mortar and pestle. Total RNA was extracted using the NucleoSpin RNA Plant Kit (Macherey Nagel) according to the manufacturer instruction. cDNA synthesis was carried out with the iScript Ready-to-Use cDNA Supermix (Bio-Rad Laboratories, Inc) according to the manufacturer instructions. RT- qPCR was performed with SsoAdvanced Universal SYBR(r) Green Supermix (Bio-Rad Laboratories). Quantification of transcript levels were performed on a CFX96 Real-Time Detection System (Bio-Rad Laboratories, Hercules, CA) with primers shown in Table S1. Reference genes were MtTCTP and ACTIN11 commonly used in the laboratory for M. truncatula qRT-PCR analysis (Zinsmeister et al., 2016). The relative expression (RE) was normalized with the geometric mean of the 2 reference genes previously mentioned and was calculated according to the following formula: $P E=2^{\wedge} \Delta^{\prime \prime} \tau$ (where $\Delta^{\prime \prime} \tau=$ geometric mean of the $\mathrm{Ct}$ reference genes - $\mathrm{Ct}$ target gene) with $\mathrm{Ct}$ is the value of the detection cycle of the transcript. Each point represents the average of three independent biological replicates of 30 seeds each.

\section{RNAseq and data analysis}

For RNAseq analysis, RNA was extracted from three replicates of 25 mature seeds of wild type, Mthsfa9-1 and Mthsfa9-2 alleles that were grown together. RNAseq was outsourced to BGI Genomics (Shenzhen, China). RNA quality was checked with an Agilent 2100 Bio analyzer (Agilent RNA 6000 Nano Kit). RNA was sequenced using the DNBseq platform, generating an average $24 \mathrm{M}$ reads per sample. The sequencing reads which containing low-quality, adaptor-polluted and high content of unknown base $(\mathrm{N})$ reads were removed and mapping of the clean reads was performed against the Mt4.0 genome. Total mapping ratio was $90 \%$, and unique mapping ratio $78 \%$. DEGs (differential expressed genes) between samples were determined using DEseq2 algorithms. Genes were considered differential when the adjusted Pvalue was $<0.05$ and log2FoldChange of 1 . On the DEGs, GO (http://www.geneontology.org/) and KEGG (https://www.kegg.jp/) enrichment analysis of annotated different expression gene was performed by Phyper (https://en.wikipedia.org/wiki/Hypergeometric_distribution) based on Hypergeometric test. The significant levels of terms and pathways were corrected by $\mathrm{Q}$ value with a rigorous threshold (Q value [?] 0.05) by Bonferroni. RNAseq data were deposited in the NCBI Gene Expression Omnibus database (accession number GSE98199 and GSExxxx).

\section{Transcriptomic analysis of Medicago truncatula hairy roots overexpressing 35S::MtHSFA9::GFP compared to 35S::GUS::GFP}


For ectopic overexpression of MtHSFA9 ,35S::MtHSFA9::GFP/35::GUS and the control (plasmid containing the GUS gene alone) plasmids were obtained by the gateway cloning method according to Verdier et al (2013) and used forAgrobacterium rhizogenes transformation for subsequent hairy root transformation in Medicago truncatula following the protocol of (Boisson-Dernier et al. 2001). MtHSFA9 and GUSamplicons were obtained by PCR using primers listed in Table S1 and cloned in pK7GW2D,1 (Karimi, Inzé \& Depicker 2002) containing a $35 \mathrm{~S}$ cauliflower mosaic virus promoter and GFP reporter that was used as transformation marker. Seedlings were grown in square Petri dishes with Fahraeus medium two months at $20^{\circ} \mathrm{C}, 16 \mathrm{~h}$ light, at an angle of $45^{\circ}$. Transformed roots were selected by GFP fluorescence under UV microscope (stereomicroscope, Olympus U-RFL-T) and harvested in liquid nitrogen. RNA was extracted from three replicates of similar amounts of roots using Nucleospin Extract II, Total RNA Purification Kit (Macherey Nagel). RNA amplification, labeling, and hybridization of Nimblegen Medtr_v1.0 12x135K arrays were performed according to Terrassonet al. (2013). Three biological replicates were analyzed per comparison using the dyeswitch method, and statistical analysis on the gene expression data was performed according to Verdier et al. (2013). Transcriptome data has been submitted to the Gene Expression Omnibus as GSExxxxx.

\section{Phylogenetic analysis}

The 14 HSFA amino acid sequences of Arabidopsis were retrieved from the TAIR data base. The amino sequence of MtHSFA9 was blasted against the legume transcription factor data base LegumeTFDB (http://legumetfdb.psc.riken.jp/) containing sequences from soybean, M. truncatula and Lotus japonica . The resulting gene list was manually curated to retain only orthologs of the HSFA family. Since the Medicago gene was originally annotated as HSA2.2, we added the amino sequence from two HSFA2 genes already characterized in the literature, TsHSFA2d and OsHSFA2d (Chauhanet al. 2011; Chauhan, Khurana, Agarwal, Khurana \& Khurana 2013). This orthologous gene set together with HaHSFA9 was used to construct a phylogenetic tree using neighbour-joining algorithm from the MEGA7.0 software (Kumar, Stecher \& Tamura 2016).

\section{Yeast-One Hybrid}

Fragments of 60 bp regions of promotors of MtNCED genesMedtr5g025230, Medtr5g025250, Medtr5g02527, predicted to contain a putative binding site for MtHSFA9(http://plantpan2.itps.ncku.edu.tw) (Table S1) were cloned into pBait-AbAi vector as bait constructs. The coding sequence of MtHSFA9 was cloned into the pGADT7-Rec AD cloning vector as prey construct. The empty pGADT7-Rec plasmid was used as negative control. Transformation and screening process were carried out on the media containing SD-Leu and SD-Leu $+\mathrm{AbA}$ antibiotic, respectively, according to the manufacturers' instructions (www.clontech.com).

\section{RESULTS}

\section{Identification of the seed-specific MtHSFA9 in M. truncatula as the ortholog of HaHSFA9}

To identify transcription factors (TF) that underly the regulation of the acquisition of desiccation tolerance (DT) and longevity, we performed a detailed temporal transcriptome study during seed development (Verdier et al. 2013). The construction of a gene co-expression network of genes and seed specific transcription factors that correlated with DT and longevity inferred a number of TFs as putative regulators of late maturation/longevity genes (Verdier et al. 2013). Detailed characterization of this network identified a heat shock transcription factor (Medtr4g126070/HSFA) as a central hub of the module, with the highest betweenness centrality (Fig. 1a, Data S1). In addition, this HSFA was one of the nodes with the closest centrality and number of connected nodes, with only Medtr1g023170, a DREB2D TF, being more connected (Data S1).

A blast of the $M$. truncatula sequence against Arabidopsis TAIR11 revealed homology with three AtHSFA: At2g26150/HSFA2 (7.55e-75), At3g22830/HSFA6B (1.25e-72) and AT5G54070/AtHSFA9 (7.17e-72). To analyze to which group the $M$. truncatula HSFA belongs, a phylogenic tree was constructed using homologous HSFA protein sequences retrieved from M. truncatula, Glycine max and Lotus japonica databases (Fig. 1b). The phylogenetic reconstruction was performed using maximum parsimony and identified Medtr4g126070 as part of a subgroup containing AtHSFA9, HaHSFA9, GmHSFA9.1 and GmHSFA9.2 (Fig. 1b). We therefore 
referred to the gene as MtHSFA9 . Since the Arabidopsis and sunflower HSFA9 are specifically expressed in seeds, we investigated the expression patterns of MtHSA9 and the other HSFA genes (Fig. 1c). MtHSFA9 is predominantly expressed in seed tissues, with the highest transcript levels at the end of seed maturation (36 days after pollination (DAP)) (Fig. 1c, Fig. S1a). A moderate level of transcript was also detected in shoots submitted to severe drought.

\section{Identification of downstream targets of MtHSFA9}

As a first proxy to understand the function of MtHSFA9, putative target genes were identified using ectopic expression in hairy roots of $M$. truncatula followed by transcriptome analysis. A total of 420 transcripts were significantly up-regulated, and 253 transcripts downregulated in the $35 S:: M t H S F A 9:: G F P$ hairy roots compared to roots transformed with a control plasmid $(\mathrm{p}<0.01)$ (Fig. 2a). A total of 11 HSP and sHSP, and three HSF were amongst the highest differentially expressed genes (Data S2). Next we identified two knock out lines in the MtHSFA9 gene, Mthsfa9-1 and Mthsfa9-2 with TNT1 insertions at position 6 and 46 respectively from ATG (Fig. S1b). For each line an associated with type was also selected, corresponding to the line without an insertion at the MtHSFA9 genes at the corresponding position. RNA seq analysis was performed on Mthsfa9 mutants and identified 2323 down-regulated and 2408 up-regulated transcripts in mature mutant seeds compared to wild type seeds (Fig. 2a, Fig. S1c, Data S3). A Venn diagram shows the overlap between the data set from the hairy roots and deregulated genes in the mutant seeds compared to their respective controls (Fig. 2a, Dataset S2). A total of 95 genes were identified as putative targets of $M t H S F A 9$, with 67 positively regulated and 28 negatively regulated. RT-qPCR validated the reduced transcript level in mature Mthsfa9 seeds for MtHSP70 , MtHSP18.2 and MtHSP17.5 compared to the wild type and associated wild type seeds (Fig $2 \mathrm{~b}-\mathrm{d}$ ). Several putative target genes positively regulated by MtHSFA9 encoded members of the class I and class II HSP family (5), three HSP70 and two additional HSF, HSFB2A and $H S F A 2$. Projection of these putative targets on the co-expression gene network identified 10 genes, out of which eight were directly connected to MtHSFA9 and one connected with MtHSFB1, another HSF that was deregulated when MtHSFA9 is mutated (Fig. S2a). Other putative targets positively regulated by MtHSFA9 and known to be involved in the regulation of the heat shock response were the co-chaperone regulator peptidyl-prolyl cis-trans isomerase FKBP65/ROF1 (Meiri \& Breiman 2009) and BCL-2-associated athanogene6/BAG6 (Nishizawa-Yokoi, Yoshida, Yabuta \& Shigeoka 2009). Also, genes encoding for enzymes involved in the synthesis of raffinose family oligosaccharide (RFO seed imbibition 2, a raffinose synthase and galactinol synthase). Analysis of the soluble sugar content in mature seeds of the Mthsfa9 mutants revealed that glucose contents were higher compared to wild type and associated wild type seeds (Fig. 2e), whereas verbascose content was lower (Fig. 2h). No significant difference was detected for sucrose and stachyose, the major soluble sugars in mature seeds (Fig. 2f-g).

Further identification of the biological functions of the downstream pathways modified by MtHSFA9 was investigated by KEGG mapping and Gene Ontology (GO) enrichment (Fig. 2i and Fig. S2b). The main KEGG pathways that were significantly deregulated in the Mthsfa9 seeds were related to biosynthesis of secondary metabolites (flavonoid biosynthesis), carotenoid biosynthesis, porphyrin and chlorophyll metabolism, glutathione metabolism, sphingolipid metabolism, carbon fixation and carbon metabolism (Fig. 2e). Enriched GO categories that were complementary to the KEGG mapped pathways included transport, DNA replication and initiation, sulfur compounds and response to abiotic stimulus (containing all the HSP/HSF) (Fig. S2b).

\section{HSFA9 is not implicated in seed longevity but improves thermotolerance during wet storage}

The main function identified for the sunflower homologue of HSFA9 is a role in seed deterioration during storage (Prieto-Dapena et al. , 2006; Tejedor-Cano et al. , 2010). To investigate if MtHSFA9 is involved in seed longevity, seeds of wild type and Mthsfa9mutants were produced under standard growth conditions, and viability loss was followed during storage using moderate accelerated storage conditions ( $75 \% \mathrm{RH}$ and $35^{\circ} \mathrm{C}$ ). No difference could be detected in longevity, both the survival curves and the P50, determined as the storage time needed for the seed population to lose $50 \%$ of their viability, were similar between wild types and Mthsfa9 mutants (Fig. 3a). Two additional cultures were grown and storage experiments were 
conducted on the harvested seeds, and this confirmed the absence of a perturbed longevity phenotype of the Mthsfa9 mutants (Fig. S3a,b).

Next, we hypothesized that MtHSFA9 might have a role in the regulation of longevity when the mother plants are grown under high temperature conditions. Indeed in $M$. truncatula, high temperature $\left(26^{\circ} \mathrm{C}\right)$ was shown to reduce longevity in the A17 genotype (Righetti et al., 2015). To test this hypothesis, wild type and mutant seeds were produced at $26^{\circ} \mathrm{C}$, which shortened seed development compared to $20^{\circ} \mathrm{C}$ for all genotypes, with pod abscission occurring at $552-575^{\circ}$ days at $26^{\circ} \mathrm{C}$ compared to $731-748^{\circ}$ days at $20^{\circ} \mathrm{C}$. Seed filling was significantly impacted by the high temperature, in a similar manner for the Mthsfa9 mutants and wild type (Fig. S4a). Longevity was significantly higher for all genotypes when seeds were produced at $26^{\circ} \mathrm{C}$ compared to $20^{\circ} \mathrm{C}$ (Fig. S4b and c). However, no significant differences were observed between wild type and Mthsfa 9 seeds. We then examined if high temperature affected transcript levels of putative target genes of MtHSFA9 (Fig. S4d-f). Whereas $\mathrm{HSP}^{70}$ transcript levels were lower in wild type seeds grown at $26^{\circ} \mathrm{C}$ compared to $20^{\circ} \mathrm{C}, M t H S P 17.5$ and $M t H S P 18.2$ transcripts significantly increased during the high temperature treatment (Fig. S4e-f). Yet, transcript levels of these three genes were not significantly affected by heat in the Mthsfa 9 mutants compared to the wild type seeds.

Since no longevity phenotype could be observed in the Mthsfa9seeds, we hypothesized that the difference between our study and previous works (Prieto-Dapena et al. , 2006; Tejedor-Cano et al. , 2010) might originate from differences in the seed aging protocols. To verify this, we used the Controlled Deterioration Test (CDT) protocol by equilibration at $100 \% \mathrm{RH}$ and $40^{\circ} \mathrm{C}$, that we refer to as 'wet aging'. Under these conditions, seeds of the Mthsfa9mutants lost their viability much faster than wild type seeds, showing over three-fold reduction in lifespan compared to wild type seeds (Fig. 3b). We further investigated the effect of $\mathrm{RH}$ on seed lots that were after-ripened for 7 months and submitted to the same storage temperature $\left(35^{\circ} \mathrm{C}\right.$ ) but different $\mathrm{RH}$ (Fig. S5a). Storage of seeds at $100 \% \mathrm{RH} / 35^{\circ} \mathrm{C}$ or $85 \% \mathrm{RH} / 35^{\circ} \mathrm{C}$ also showed a faster deterioration phenotype for the Mthsfa 9 mutants, evident from a reduction in germination speed and final germination percentage (Fig. S5b,c). Water contents at the different storage conditions after 56 days of equilibration were $0.14 \mathrm{~g} \mathrm{H}_{2} \mathrm{O} / \mathrm{g}$ DW at $75 \% \mathrm{RH}, 0.18 \mathrm{~g} \mathrm{H}_{2} \mathrm{O} / \mathrm{g} \mathrm{DW}$ at $85 \% \mathrm{RH}$ and $0.84 \mathrm{~g} \mathrm{H}_{2} \mathrm{O} / \mathrm{g}$ DW at $100 \% \mathrm{RH}$ regardless of the genotype (Fig. S5d). This indicated that elevating the water contents to allow metabolism activates protective or repair processes governed by HSFA9 that do not occur during dry aging.

To verify whether these findings were only applicable to HSFA9 from $M$. truncatula, we isolated an Arabidopsis T-DNA insertion mutant in which transcript level of HSFA9 (At5g54070) was strongly reduced (Fig. S6a,b). The transcripts of two putative targets, HSP17.4 and HSP17.6 (Almoguera et al. 2002; Kotaket al. 2007) were significantly decreased in mature mutant seeds, thereby demonstrating that HSFA9 is a transcriptional activator of these two HSPs in Arabidopsis seeds (Fig. S6c). Like in M. truncatula, mature seeds of the wild type and Athsfa9 mutants lost their viability at a comparable rate during storage at $75 \% \mathrm{RH}$ and $35^{\circ} \mathrm{C}$ (Fig. 3c). In contrast, when seeds were submitted to the CDT protocol, Athsfa9 mutants lost their viability much faster than wild type (Fig. 3d). This suggests that HSFA9 or downstream targets begin to function in the seeds when water content increases through equilibration between $75 \%$ and $85 \% \mathrm{RH}$.

\section{MtHSFA9 plays a role in the release of dormancy during late maturation}

When the viability of the seed lots was tested, we noticed that promptly after harvest, seeds took a very long time to germinate. Since $M$. truncatula seeds exhibit physiological dormancy, apparent from a strong reduction in germination speed that is released during after-ripening (Bolingue et al, 2010), we decided to further investigate this behaviour. Seeds of the different genotypes were harvested at pod abscission and dried for three days at $43 \% \mathrm{RH}$ to homogenize the water content before imbibition. Whereas wild type seeds germinated within 2-3 days, seeds from Mthsfa9 lines germinated at around 8-10 days (Fig. 4a). Stratification for $3 \mathrm{~d}$ at $4^{\circ} \mathrm{C}$ drastically increased the germination speed of the mutants, and only a very slight difference was still apparent between wild type and Mthsfa9 lines (Fig. 4b). During after-ripening, the difference in germination speed between the mutants and wild type seeds remained significant up until 5 months, but the relative rate of after-ripening did not differ between the genotypes (Fig. 4c). 
Since MtHSFA9 transcript levels increased around 20 DAP and reached a maximum at 36 DAP (Fig. S1a), dormancy levels were determined as mean germination time in freshly harvested seeds from 20 DAP until pod abscission (Fig. 4d). At 20 DAP, prior to the acquisition of dormancy (Bolingue, Vu, Leprince \& Buitink 2010), no difference was found in the dormancy levels between the genotypes (Fig. S7a). Significant differences were observed at final maturation, with the highest difference prior to full maturation drying, at 40 DAP (Fig. 4d, Fig. S7b). Absence of MtHSFA9 doubled the mean germination time from 35 to 70d, indicating that MtHSFA9 regulates the depth of dormancy acquisition during late maturation. Seed development at $26^{\circ} \mathrm{C}$ did not change the dormancy level in the Mthsfa9 mutants compared to wild type (Fig. S7c). Freshly harvested Arabidopsis hsfa9 seeds showed reduced germination but this was comparable to wild type seeds (Fig. S7d). After-ripening increased the germination percentage and speed, but no difference could be detected between the Athsfa9 and wild type seeds (Fig. S7d).

\section{HSFA9 regulates dormancy via modulation of ABA metabolism and signaling}

To understand the increased dormancy phenotype of the Mthsfa 9 seeds, we searched for evidence whether ABA or GA signaling pathways were affected by comparing the transcriptomes of Mthsfa 9 seeds and hairy roots ectopically expressing MtHSFA9 . For ABA, five genes were differentially expressed in both analyses. Three genes had higher transcript levels in the Mthsfa9 mutants, and were downregulated when MtHSFA9 was expressed in the hairy roots: anindole-3-acetaldehyde oxidase (AAO, Medtr5g087410) that is involved in ABA biosynthesis, PYL/PYR6 (Medtr5g083270), an ABA receptor, and HVA22 , a marker gene of ABA response (Data S2 and Fig. 5a). In addition, a cytochrome P450 (CYP707A Medtr8g072260), involved in ABA degradation, was also differentially expressed in both studies. We also noticed that one of the most significantly overrepresented GO/KEGG category in the Mthsfa9 mutants was carotenoid metabolism (Fig. $2 \mathrm{i}$, Fig S2b). A closer look at the genes in this pathway revealed that those involved in ABA biosynthesis were upregulated, including a zeaxanthin epoxidase $(Z E P)$ and three 9-cis-epoxycarotenoid dioxygenase (NCED) , whereas two other $C Y P^{7} 70^{7} A$ genes were downregulated (Fig. 5a), suggesting that MtHSFA9 could regulate ABA homeostasis. RT-qPCR confirmed the differential expression of these genes in mature seeds from wild type and Mthsfa9 mutants from a different harvest (Fig. 5b-d). For GA, the comparison of both transcriptomes revealed the biosynthesis gene gibberellin 3-beta- dioxygenase 1 (GA3OX1) being downregulated in the Mthsfa 9 seeds and upregulated in the transgenic hairy roots. In addition, several other genes related to GA metabolism were deregulated in the Mthsfa9 seeds, namely two orthologs of gibberellin 2-beta-dioxygenase 2 (GA2OX2) involved in GA inactivation that were upregulated in the mutants, and one gene involved in GA biosynthesis, gibberellin 20 oxidase 2 (GA20OX2), that was downregulated. A GA receptor, GID1 was also found to have reduced transcript levels in the Mthsfa 9 seeds. Another putative link between GA is suggested by the deregulation of the homolog of the protein REVEILLE1 (RVE1) both in hairy roots and Mthsfa9 seeds (Data S2). This gene was shown to directly inhibitsGA3OX2 transcription in Arabidopsis and affect dormancy (Yang, Jiang, Liu \& Lin 2020). All these results are consistent with the dormancy phenotype in Mthsfa9 seeds.

Considering the higher transcript levels of ABA biosynthesis genes and decreased levels of ABA degradation genes, ABA content was determined in mature seeds. A significant increase in ABA was observed in the mature Mthsfa9 seeds compared to the wild type seeds, with an increase from 317 to 511 and $576 \mathrm{fmol} / \mathrm{g}$ DW for Mthsfa9-1 and Mthsfa9-2, respectively (Fig. 6a). To investigate whether the dormancy phenotype of Mthsfa 9 seeds was due to further ABA biosynthesis during imbibition, freshly harvested seeds were imbibed in the ABA inhibitor fluridone. For all genotypes, fluridone promoted germination speed. However, compared to their respective mock controls, the impact of the fluridone treatment on the time to $50 \%$ germination (T50) was not significantly different between Mthsfa9 and wild type seeds (Fig. 6b). This suggests that dormancy in Mthsfa9 seeds could not be explained by de novo ABA synthesis during imbibition but rather originates from differences induced during seed maturation. Further evidence that the dormancy phenotype of Mthsfa 9 is related to the maturation program and not activated during imbibition comes from the expression of the three hormone metabolism genes that were deregulated in the Mthsfa9 mutants:MtNCED4, MtCYPro7A and MtGA3OX1. These three genes showed clear differential expression profiles during imbibition between dormant and non-dormant wild type seeds (Fig. S9a-c). However, imbibition of Mthsfa9 seeds did not show 
this typical dormancy profile (Fig. S9d-f).

Besides differences in ABA metabolism, several other ABA signaling genes were deregulated in the Mthsfa 9 mutants (ABA receptor PYL6, protein phosphatase 2C, PP2C, BURP domain protein RD22 , HVA22 protein; Fig. 5a) suggesting that Mthsfa9 seeds could exhibit altered ABA sensitivity during imbibition. This was tested by incubating after-ripened seeds in increasing ABA concentrations (Fig. 6c). Mutant seeds showed hypersensitivity when imbibed in darkness compared to wild type (Fig. 6c).

Physiological dormancy can be imposed by the embryo or the surrounding tissues (testa, endosperm) or combinations of these tissues, and their sum and interaction determine the depth of dormancy (Chahtane, Kim \& Lopez-Molina 2016; Penfield 2017). To evaluate whether the dormancy phenotype of the Mthsfa9 mutants came from the embryo or the covering structures, isolated embryos were incubated in water at $25^{\circ} \mathrm{C}$ and radicle growth was monitored (Fig. 6d). For all genotypes, embryo radicles grew faster in coat-less seeds, as previously observed (Bolingue et al. 2010). However, the time for the radicle to initiate growth was longer for the isolated Mthsfa 9 embryos compared to the wild type embryos and the final percentage of seeds with radicles that had grown was smaller in Mthsfa9 compared to wild type (Fig. 6d, Fig. S7e). Thus, the difference in germination/growth in the mutants remains visible without the surrounding layers and appears to reside mostly in the embryo.

The heat shock elements (HSE) that HSFA9 binds to has been shown to bind a modified HSE (Carranco, Almoguera \& Jordano 1999). It was suggested that the specific sequence of this heat stress cis-element is crucial for HsfA9 promoter selectivity, and that this selectivity could involve preferential transcriptional activation following DNA binding. We investigated if a similar pattern could be observed for the promotors of the putative MtHSFA9 targets, starting with the two most likely targets: HSP17.5 and MtHSP18.2 (Fig. 2b, c; Kotak et al., 2007). The promotor of HSP17.5 contained one perfect HSE (Table S2, Guo et al. , 2008). HSP18.2 did not contain any perfect HSE, and neither of the two promotors contained the modified HSE detected for HaHSAF9. For the other 22 hormone-related genes that were deregulated in the Mthsfa9mutants (Fig. 5; Table S2), all HSE sites contained at least 2 out of 9 mismatches, but no common sequence could be found that could be tested as a putative binding site for MtHSFA9. However, for six genes (MtPYL6, MtPP2C, $A A O, N C E D, H V A 22$ and Ga20Ox ), a similar modified sequence GAAnnTTXnnXAA was identified, and three of these genes also show deregulation in the hairy root ectopically expressing MtHSFA9 (Fig. 5). Since $M t N C E D 4$ was one of the most differentially expressed genes (Fig. 5a), a yeast one hybrid assay was carried out to determine if MtHSFA9 could bind to the putative HSE present in the promoter region of this gene (Fig. S8). No interaction could be detected, suggesting that this gene is either not under the direct regulation of MtHSFA 9 or that other parts of the promotor are needed for the interaction.

\section{DISCUSSION}

HSFA gene family members play a crucial role in plant response to several abiotic stresses by regulating the expression of stress-responsive genes including heat shock proteins (Ohama et al. 2017; Jacob et al. 2017). A peculiar HSF, HSFA9, has been reported in several species with an expression being restricted to seeds, more precisely increasing during the final part of seed development (Almoguera et al. 2002; Kotak et al. 2007; Liet al. 2015). This work tested whether a putative homologue of HSFA9 from M. truncatula, a hub gene involved in seed maturation (Fig. 1) plays a role in seed longevity, defined as the time seeds remain viable after dry storage. We demonstrated that at mild storage conditions, hsfa 9 mutants of both M. truncatula and Arabidopsis did not show an altered longevity phenotype, but more humid and hot storage conditions revealed a strong decrease in seed viability (Fig. 3), the later corroborating with earlier studies using heterologous ectopic expression in tobacco (Prieto-Dapena et al. , 2006; Tejedor-Cano et al. , 2010). Further physiological characterization of Mthsfa9 mutant seeds combined with transcriptomic analysis revealed that MtHSFA9 is a regulator of the depth of seed dormancy during seed maturation via the modulation of ABA homeostasis and signalling.

Our data show that HSFA9 does not play a role in seed longevity when mild storage conditions are used. Only when moisture in the seeds was increased by the CD test, seed viability was lost at a faster rate in the 
hsfa9 mutants. It is well known that the water content and temperature combination in the seeds during storage will determine the rate and type of ageing reactions (Buitink \& Leprince 2008; Ballesteros \& Walters 2019; Zinsmeister et al. 2020). In our system, seeds were exposed to mild storage conditions $\left(75 \% \mathrm{RH}, 35^{\circ} \mathrm{C}\right)$ whereas transgenic tobacco seeds over-expressing HaHSFA9 were exposed to $42^{\circ} \mathrm{C}$ and $100 \%$ RH (PrietoDapena et al. 2008). Under these conditions, seeds imbibe and resume metabolism while tolerating the heat stress, considering that the onset of respiration is at 90-92\% RH for a wide range of species (Vertucci \& Roos 1990). Consistent with this, OsHSP18.2 gene expression increased 60 fold when rice seeds were stored for 6 days at $45^{\circ} \mathrm{C}, 100 \% \mathrm{RH}$ (Kaur et al. 2015). These storage conditions are far from ours where the seed water content was still low enough to prevent resumption of metabolism during storage. There are increasing concerns with regard to the similarity between accelerated aging conditions such as the CD test and so called natural/ambient aging conditions (Schwember \& Bradford 2010; Nagelet al. 2015; Zinsmeister et al. 2016; Roach, Nagel, Börner, Eberle \& Kranner 2018; Hay et al. 2018). Here we demonstrate that at least for the hsfa9 phenotype, results obtained by these methods are not comparable and it is evident that care has to be taken in extrapolating accelerated aging conditions to dry storage. Hay and colleagues (Hay et al. 2018) recommended that seed ageing protocols should be designed based on the potential downstream use of the findings and the biological significance of longevity. Therefore, the role of HSFA9 might be confined to situations in nature, when seeds are buried in the soil and submitted to rehydration-dehydration cycles rather than situations where seeds are stored in the dry state for gene banking purposes. It would be interesting to know if under more moderate soil temperatures $\left(10-25^{\circ} \mathrm{C}\right)$, HSFA9 still exerts its protective function on seed viability. Some suggestion that this might be the case comes from the increased tolerance against osmotic stress during imbibition in tobacco seedlings over-expressing HaHSFA9 (Prietro-Dapena et al., 2008) and in Arabidopsis seeds over-expressing a sHSP of rice (Kaur et al. 2015). The most plausible downstream genes regulated by HSFA9 that confer the protection during wet and hot storage are likely to be HSP, since their modulation correlates with seed viability (Prieto-Dapena et al. 2006; Tejedor-Cano et al. 2010; Kaur et al. 2015). Our transcriptome data is consistent with this as MtHSFA9 modulated the expression several (s)HSP (Data S2). Considering the chaperone function of sHSP, the absence of decreased longevity in hsfa9 seeds raises the intriguing possibility that protein aggregation or misfolding might not occur in the dry state where molecular motion is strongly constrained (Buitink 2000, Ballesteros and Walters 2019). Besides sHSP, our transcriptomic data revealed that several components of the chaperone signalling involved in thermotolerance are being deregulated, namely three HSP70, two additional HSF, HSFB2A and HSFA2, as well as ROF1 and BAG6. Interestingly, HSFA2 is a direct target of the master regulator HSFA1 and essential in the induction of the heat shock response (Charng et al. ,2007; Jacob et al. , 2017; Ohamaet al. , 2017). Considering that HSFA2 is induced by ABA (Huang et al. 2016), the increased ABA content in developing Mthsfa9 seeds could have induced HSFA2 and its targets. In Arabidopsis, ROF1 is thought to prolong the thermotolerance by interaction with Hsp90.1 and sustaining the level of HsfA2-regulated small HSPs (Meiri and Breiman, 2009). The ortholog of BAG6, a co-chaperone that also improves basal thermotolerance in Arabidopsis through the regulation of sHSP transcriptional cascade (Echevarría-Zomeño et al. 2016), was co-expressed withMtHSFA9 $(\mathrm{r}=0.88)$ in seeds. BAG6 is also a target of HSFA2 (Nishizawa-Yokoi et al. , 2009).

Our results show that freshly harvested seeds of Mthsfa9 mutants germinated much slower than wild type seeds (Fig. 4). Freshly harvested wild type seeds at 40 DAP took $35 \mathrm{~d}$ for half of the population to germinate, whereas Mthsfa9 seeds to double the time, $69 \mathrm{~d}$. That this was due to a dormancy phenotype was confirmed by the removal of the phenotype by either stratification or after-ripening, leading to germination within a day (Fig. 3). Few studies link HSF or sHSPs to dormancy. A proteome study on wild Lolium rigidum (annual ryegrass) subpopulations selected for low and high levels of primary dormancy revealed that highdormancy seeds showed higher abundance of sHSP than the low-dormancy seeds, and high-dormancy seeds were more tolerant to high temperatures imposed at imbibition (Goggin, Powles \& Steadman 2010). Our data provide direct evidence suggesting that the role of HSFA9 is to regulate the depth of seed dormancy that is acquired during late maturation (Fig. 4d). In Arabidopsis, the depth of dormancy is regulated by DOG1 (Footitt et al. 2020). Interestingly in this species, HSFA9 and its targets were downregulated in thedog1-1 mutant and it was suggested that DOG1 activates this transcriptional cascade independently of ABI3 (Dekkers et al.2016). Another putative cascade in which HSFA9 could be a player in regulating depth of 
dormancy is via the endosperm expressed transcription factors ICE and ZHOUPI that act during maturation to determine the depth of primary dormancy (MacGregor et al. 2019). During late embryogenesis and in mature seeds, ICE1 inhibits the expression of the transcription factor $A B I 3$, which itself is a central player in the formation of dormant seeds (Giraudat et al.1992). In Arabidopsis, ABI3 regulates HSFA9 (Kotak et al. 2007), and MtHSFA9 transcripts are also downregulated in developing Mtabi3 mutants (Verdier et al. 2013). Interestingly, ICE has been found to interact with a complex including HSFA1d (Bulgakovet al. 2019). Here, we did not find a difference in dormancy of mature Arabidopsis hsfa 9 mutants compared to wild type (Fig. S7). However, Arabidopsis Col-0 accession seeds were not very dormant under our growth conditions, and considering that the differences in dormancy between Mthsfa 9 and wild type were highest prior to maturation drying (Fig. 4d), our study might not have been able to reveal potential differences. Alternatively, considering the apparent absence ofDOG1 in M. truncatula genome, MtHSFA9 might play a more preponderant role in dormancy in M. truncatula than in Arabidopsis.

The dormancy phenotype of the Mthsfa9 mutants can be explained by an increase in ABA content in mature seeds together with an increased ABA sensitivity during imbibition (Fig. 6). This phenotype was accompanied by the deregulation of a number of genes involved in ABA homeostasis, as well as the upregulation of a putative ABA receptor (Fig. 5). Whereas it is known that ABA acts upstream of HSFAs and induces its expression (Kotak et al. 2007; Huang et al.2016; Ohama et al. 2017), downstream target genes of HSFAs have so far not been implicated in ABA or GA signalling. The only suggestion that ABA signalling might be affected downstream of HSFAs comes from Huang et al (2016). These authors showed that overexpression of HSFA6 induced hypersensitivity to ABA, which is opposite of our findings. In addition, the expression of RD22 , a drought-responsive marker gene mediated by the transcription activators MYB/MYC in an ABA-dependent manner (Abe et al. 2003) was unaffected in HSFA6b mutants but upregulated in the Mthsfa9 mutants (Fig. 5). The ortholog of MYC2 in M. truncatula (Medtr5g030430.1) was also upregulated in the Mthsfa9mutants (Data S3). Amongst the differentially expressed genes both in Mthsfa9 mutants and in hairy roots via ectopic expression of MtHSFA9 (Fig. 5), we identified several genes that show a typical dormancy-related expression profile during imbibition in dormant versus fully after-ripened $M$. truncatula seeds, MtNCED4, MtCYP707A and MtGa2OX2 (Fig. S9). MtCYP707A(Medtr8g72260) is an ABA 8'-hydroxylase showing highest homology withAtCYP707A1, a gene that regulates ABA levels and dormancy during maturation (Okamoto et al.2006). Another link between HSFA9 and dormancy could be via the regulation of GA homeostasis. This is supported by the observation that GAox genes were deregulated in mature dry Mthsfa9 seeds. Also, RVE1 was upregulated in the mutants and downregulated in the hairy roots (Data S2). In Arabidopsis, overexpression of RVE1 lead to increased dormancy (Yang et al.2020). This gene directly inhibits GA3OX2 transcription, suppressing GA biosynthesis. We were not able to alter germination speed in Mthsfa9 seeds using paclobutrazol. While this supports a role of MtHSFA9 during maturation rather than imbibition, we cannot exclude that this GA biosynthesis inhibitor does not penetrate the seed coat (Bolingue et al. 2010). While this work suggests that MtHSFA9 acts as a regulator of dormancy depth, future work is needed to decipher whether HSF acts directly on genes regulating hormone metabolism and sensitivity or whether it acts indirectly through the action of specific HSPs under the control of MtHSFA9.

\section{Short legends for Supporting Information}

Figure S1. Characterization of Mthsfa9 mutants.

Figure S2 Identification and characterization of MtHSFA9 downstream (in)direct targets.

Figure S3. Survival curves of mature wild type (WT), associated WT (assocWT) and Mthsfa9 seeds from different growth environments.

Figure S4. Effect of high temperature during seed development on seed number, longevity and gene expression of HSP that are deregulated in Mthsfa9 mutants

Figure S5. Water content and germination curves after aging at different relative humidities $(\mathrm{RH})$ of mature wild type (WT), associated WT (assocWT) and Mthsfa9 seeds. 
Figure S6. Deregulation of HSFA9 and two sHSP targets in Arabidopsishsfa9 mutants.

Figure S7. Seeds of M. truncatula Mthsfa9 Tnt1 insertion mutants exhibit a dormancy phenotype.

Figure S8. MtHSFA9 does not bind to the promotor of MtNCED4.

Figure S9. Differential expression of ABA synthesis, degradation, and GA inactivation genes in dormant and non-dormant Medicago truncatulawild type and hsfag seeds.

Supplemental Data S1. Network parameters from co-expression module connecting seed-specific transcription factors to late maturation genes.

Supplemental Data S2. Differentially regulated genes in $35 S:: M t H S F A 9$ hairy roots and Tnt1 insertion mutants of

MtHSFA9

Supplemental Data S3. Differentially expressed genes in Tnt1 insertion mutants of MtHSFA9 (Medtr4g126070).

Table S1. List of primers used for experiments performed by PCR and for Yeast-One Hybrid.

Table S2. Potential heat shock element binding sites in promotors of genes that are deregulated in seeds of Mthsfa 9 mutants.

Table S3. Gene identifiers used for the maximum parsimony analysis shown in Figure 1.

\section{References}

Abe H., Urao T., Ito T., Seki M., Shinozaki K. \& Yamaguchi-Shinozaki K. (2003) Arabidopsis AtMYC2 (bHLH) and AtMYB2 (MYB) function as transcriptional activators in abscisic acid signaling. The Plant cell 15, 63-78.

Almoguera C., Prieto-Dapena P., Personat J.-M., Tejedor-Cano J., Lindahl M., Diaz-Espejo A. \& Jordano J. (2012) Protection of the photosynthetic apparatus from extreme dehydration and oxidative stress in seedlings of transgenic tobacco. PLOS ONE 7 .

Almoguera C., Rojas A., Diaz-Martin J., Prieto-Dapena P., Carranco R. \& Jordano J. (2002) A seedspecific heat-shock transcription factor involved in developmental regulation during embryogenesis in sunflower. Journal of Biological Chemistry 277 , 43866-43872.

Ballesteros D. \& Walters C. (2019) Solid-state biology and seed longevity: a mechanical analysis of glasses in pea and soybean embryonic axes. Frontiers in plant science 10, 920.

Bettey M. \& Finch-Savage W.E. (1998) Stress protein content of mature Brassica seeds and their germination performance. Seed Science Research $8,347-355$.

Boisson-Dernier A., Chabaud M., Garcia F., Becard G., Rosenberg C. \& Barker D.G. (2001) Agrobacterium rhizogenes-transformed roots of Medicago truncatula for the study of nitrogen-fixing and endomycorrhizal symbiotic associations. Molecular Plant-Microbe Interactions14, 695-700.

Bolingue W., Vu B.L., Leprince O. \& Buitink J. (2010) Characterization of dormancy behaviour in seeds of the model legume Medicago truncatula.Seed Science Research 20 , 97-107.

Buitink J. \& Leprince O. (2008) Intracellular glasses and seed survival in the dry state. 331 , 788-795.

Bulgakov V.P., Wu H.-C. \& Jinn T.-L. (2019) Coordination of ABA and chaperone signaling in plant stress responses. Trends in Plant Science 24, 636-651.

Carranco R., Almoguera C. \& Jordano J. (1999) An imperfect heat shock element and different upstream sequences are required for the seed-specific expression of a small heat shock protein gene. Plant Physiology $121,723-730$. 
Chahtane H., Kim W. \& Lopez-Molina L. (2016) Primary seed dormancy: a temporally multilayered riddle waiting to be unlocked. Journal of Experimental Botany 68, 857-869.

Charng Y., Liu H., Liu N., Chi W., Wang C., Chang S. \& Wang T. (2007) A heat-inducible transcription factor, HsfA2, is required for extension of acquired thermotolerance in Arabidopsis. Plant Physiology $\mathbf{1 4 3}$, $251 \mathrm{LP}-262$.

Chatelain E., Hundertmark M., Leprince O., Le, Gall S., Satour P., .. B Buitink J. (2012) Temporal profiling of the heat-stable proteome during late maturation of Medicago truncatula seeds identifies a restricted subset of late embryogenesis abundant proteins associated with longevity. Plant, Cell and Environment 35 , $1440-1455$.

Chauhan H., Khurana N., Agarwal P., Khurana J.P. \& Khurana P. (2013) A seed preferential heat shock transcription factor from wheat provides abiotic stress tolerance and yield enhancement in transgenic Arabidopsis under heat stress environment. PLoS ONE 8, e79577.

Chauhan H., Khurana N., Agarwal P. \& Khurana P. (2011) Heat shock factors in rice (Oryza sativa L.): genome-wide expression analysis during reproductive development and abiotic stress. Molecular Genetics and Genomics $286,171$.

Dekkers B.J.W., He H., Hanson J., Willems L.A.J., Jamar D.C.L., Cueff G., . . Bentsink L. (2016) The Arabidopsis DELAY OF GERMINATION 1 gene affects ABSCISIC ACID INSENSITIVE 5 (ABI5)expression and genetically interacts with ABI3 during Arabidopsis seed development. The Plant Journal 85 , 451-465.

Echevarria-Zomeno S., Fernandez-Calvino L., Castro-Sanz A.B., Lopez J.A., Vazquez J. \& Castellano M.M. (2016) Dissecting the proteome dynamics of the early heat stress response leading to plant survival or death in Arabidopsis. Plant, Cell 85 Environment 39 , 1264-1278.

Finch-Savage W.E. \& Bassel G.W. (2015) Seed vigour and crop establishment: extending performance beyond adaptation. Journal of Experimental Botany 67, 567-591.

Footitt S., Walley P.G., Lynn J.R., Hambidge A.J., Penfield S. \& Finch-Savage W.E. (2020) Trait analysis reveals DOG1 determines initial depth of seed dormancy, but not changes during dormancy cycling that result in seedling emergence timing. New Phytologist225, 2035-2047.

Giraudat J., Hauge B.M., Valon C., Smalle J., Parcy F. \& Goodman H.M. (1992) Isolation of the Arabidopsis ABI3 gene by positional cloning. The Plant cell $4,1251-1261$.

Goggin D.E., Powles S.B. \& Steadman K.J. (2010) Selection for low or high primary dormancy in Lolium rigidum Gaud seeds results in constitutive differences in stress protein expression and peroxidase activity. Journal of Experimental Botany 62, 1037-1047.

Graeber K., Nakabayashi K., Miatton E., Leubner-Metzger G. \& Soppe W. (2012) Molecular mechanisms of seed dormancy. Plant, Cell \& Environment 35, 1769-1786.

Guo L., Chen S., Liu K., Liu Y., Ni L., Zhang K. \& Zhang L. (2008) Isolation of heat shock factor HsfA1abinding sites in vivo revealed variations of heat shock elements in Arabidopsis thaliana. Plant 85 Cell Physiology 49, 1306-1315.

Hay F.R., Valdez R., Lee J.-S., Sta Cruz P.C. \& Sta. Cruz P.C. (2018) Seed longevity phenotyping: recommendations on research methodology. Journal of Experimental Botany 70 , 425-434.

Huang Y.-C., Niu C.-Y., Yang C.-R. \& Jinn T.-L. (2016) The heat stress factor HSFA6b connects ABA signaling and ABA-mediated heat responses.Plant Physiology 172, 1182-1199.

Jacob P., Hirt H. \& Bendahmane A. (2017) The heat-shock protein/chaperone network and multiple stress resistance. Plant Biotechnology Journal 15, 405-414. 
Karimi M., Inze D. \& Depicker A. (2002) GATEWAY vectors for Agrobacterium-mediated plant transformation. Trends in Plant Science 7, 193-195.

Kaur H., Petla B.P., Kamble N.U., Singh A., Rao V., Salvi P., .. Majee M. (2015) Differentially expressed seed aging responsive heat shock protein OsHSP18.2 implicates in seed vigor, longevity and improves germination and seedling establishment under abiotic stress.Frontiers in Plant Science 6 , 713.

Kotak S., Vierling E., Baumlein H. \& Von Koskull-Dorlng P. (2007) A novel transcriptional cascade regulating expression of heat stress proteins during seed development of Arabidopsis. Plant Cell19 , 182-195.

Kumar S., Stecher G. \& Tamura K. (2016) MEGA7: Molecular Evolutionary Genetics Analysis Version 7.0 for Bigger Datasets. Molecular Biology and Evolution 33, 1870-1874.

Leprince O., Pellizzaro A., Berriri S. \& Buitink J. (2017) Late seed maturation: drying without dying. Journal of Experimental Botany68, 827-841.

Li Z., Tian Y., Zhao W., Xu J., Wang L., Peng R. \& Yao Q. (2015) Functional characterization of a grape heat stress transcription factor VvHsfA9 in transgenic Arabidopsis. Acta Physiologiae Plantarum37 , 133.

Lima J.J.P., Buitink J., Lalanne D., Rossi R.F., Pelletier S., Da Silva E.A.A. \& Leprince O. (2017) Molecular characterization of the acquisition of longevity during seed maturation in soybean. PLoS ONE 12 .

Long R.L., Gorecki M.J., Renton M., Scott J.K., Colville L., Goggin D.E., .. F Finch-Savage W.E. (2015) The ecophysiology of seed persistence: A mechanistic view of the journey to germination or demise.Biological Reviews $90,31-59$.

MacGregor D.R., Zhang N., Iwasaki M., Chen M., Dave A., Lopez-Molina L. \& Penfield S. (2019) ICE1 and ZOU determine the depth of primary seed dormancy in Arabidopsis independently of their role in endosperm development. The Plant Journal 98 , 277-290.

Meiri D. \& Breiman A. (2009) Arabidopsis ROF1 (FKBP62) modulates thermotolerance by interacting with HSP90.1 and affecting the accumulation of HsfA2-regulated sHSPs. The Plant Journal59 , 387-399.

Nagel M., Kranner I., Neumann K., Rolletschek H., Seal C.E., Colville L., .. Borner A. (2015) Genomewide association mapping and biochemical markers reveal that seed ageing and longevity are intricately affected by genetic background and developmental and environmental conditions in barley. Plant, Cell and Environment38 , 1011-1022.

Nishizawa-Yokoi A., Yoshida E., Yabuta Y. \& Shigeoka S. (2009) Analysis of the regulation of target genes by an Arabidopsis heat shock transcription factor, HsfA2. Bioscience, Biotechnology, and Biochemistry $\mathbf{7 3}$ , 890-895.

Ohama N., Sato H., Shinozaki K. \& Yamaguchi-Shinozaki K. (2017) Transcriptional Regulatory Network of Plant Heat Stress Response. Trends in Plant Science 22, 53-65.

Okamoto M., Kuwahara A., Seo M., Kushiro T., Asami T., Hirai N., ... Nambara E. (2006) CYP707A1 and CYP707A2, which encode abscisic acid 8'-hydroxylases, are indispensable for proper control of seed dormancy and germination in Arabidopsis. Plant Physiology141, 97-107.

Penfield S. (2017) Seed dormancy and germination. Current Biology27, R874-R878.

Penfield S. \& MacGregor D.R. (2017) Effects of environmental variation during seed production on seed dormancy and germination. Journal of Experimental Botany 68, 819-825.

Personat J.-M., Tejedor-Cano J., Prieto-Dapena P., Almoguera C. \& Jordano J. (2014) Co-overexpression of two Heat Shock Factors results in enhanced seed longevity and in synergistic effects on seedling tolerance to severe dehydration and oxidative stress. BMC Plant Biology $14,56$. 
Prieto-Dapena P., Castano R., Almoguera C. \& Jordano J. (2008) The ectopic overexpression of a seedspecific transcription factor, HaHSFA9, confers tolerance to severe dehydration in vegetative organs. Plant Journal 54, 1004-1014.

Prieto-Dapena P., Castano R., Almoguera C. \& Jordano J. (2006) Improved resistance to controlled deterioration in transgenic seeds. Plant Physiology 142, 1102-1112.

Roach T., Nagel M., Borner A., Eberle C. \& Kranner I. (2018) Changes in tocochromanols and glutathione reveal differences in the mechanisms of seed ageing under seedbank conditions and controlled deterioration in barley. Environmental and Experimental Botany 156 , 8-15.

Sano N., Rajjou L., North H.M., Debeaujon I., Marion-Poll A. \& Seo M. (2016) Staying alive: Molecular aspects of seed longevity. Plant and Cell Physiology 57, 660-674.

Schwember A.R. \& Bradford K.J. (2010) Quantitative trait loci associated with longevity of lettuce seeds under conventional and controlled deterioration storage conditions. Journal of Experimental Botany $\mathbf{6 1}$, 4423-4436.

Tejedor-Cano J., Prieto-Dapena P., Almoguera C., Carranco R., Hiratsu K., Ohme-Takagi M. \& Jordano J. (2010) Loss of function of the HSFA9 seed longevity program. Plant, Cell and Environment 33 , 1408-1417.

Terrasson E., Buitink J., Righetti K., Vu B.L., Pelletier S., Zinsmeister J., ... Leprince O. (2013) An emerging picture of the seed desiccome: Confirmed regulators and newcomers identified using transcriptome comparison. Frontiers in Plant Science 4, 497.

Verdier J., Lalanne D., Pelletier S., Torres-Jerez I., Righetti K., Bandyopadhyay K., .. Buitink J. (2013) A regulatory network-based approach dissects late maturation processes related to the acquisition of desiccation tolerance and longevity of Medicago truncatula seeds.Plant Physiology 163, 757-774.

Vertucci C.W. \& Roos E.E. (1990) Theoretical basis of protocols for seed storage. Plant Physiology 94 , 1019-1023.

Wehmeyer N., Hernandez L.D., Finkelstein R.R. \& Vierling E. (1996) Synthesis of small heat-shock proteins is part of the developmental program of late seed maturation. Plant Physiology 112, 747-757.

Wehmeyer N. \& Vierling E. (2000) The expression of small heat shock proteins in seeds responds to discrete developmental signals and suggests a general protective role in desiccation tolerance. Plant Physiology 122 , 1099-1108.

Yang L., Jiang Z., Liu S. \& Lin R. (2020) Interplay between REVEILLE1 and RGA-LIKE2 regulates seed dormancy and germination in Arabidopsis. New Phytologist 225 , 1593-1605.

Zinsmeister J., Lalanne D., Terrasson E., Chatelain E., Vandecasteele C., Ly Vu B., .. L Leprince O. (2016) ABI5 is a regulator of seed maturation and longevity in legumes. Plant Cell 28, 2735-2754.

Zinsmeister J., Leprince O. \& Buitink J. (2020) Molecular and environmental factors regulating seed longevity. Biochemical Journal 477, 305-323.

\section{Figure legends}

FIGURE 1. Identification of MtHSFA9 as a seed-expressed gene. (a) Co-expression network of seed-specific transcription factors (squares) and transcripts correlating with the acquisition of desiccation tolerance and longevity. Node size and color represent the betweenness centrality and edge betweenness (yellow is low, blue is high). Recalculated from Verdier et al. (2013). (b) Maximum parsimony analysis of HSFA proteins from legumes (Glycine max,Medicago truncatula, Lotus japonica), Arabidopsis thalianaand HaHSFA9 from Helianthus annuus. Gene identifiers are provided in Table S3. Branches corresponding to partitions reproduced in less than 50\% bootstrap replicates (1000) are collapsed. The tree was obtained using the Subtree-Pruning-Regrafting algorithm using MEGA7 (Kumar et al., 2016). For soybean the two paralogs are shown when appropriate. Stars indicate the M. truncatula ortholog of HaHSFA9. (c) Heat map of the 
HSFA family genes in Medicago truncatula showing mean-centered transcript levels with high (red) to low (blue) levels. Data are from the M. truncatula gene expression atlas (https://mtgea.noble.org).

FIGURE 2 . Identification of putative targets of MtHSFA9 and downstream biological processes. (a) Venn diagram between up- or downregulated genes in hairy roots overexpressing $35 S::$ MtHSFA9::GFP::GUS compared to hairy roots transformed with35S::GFP::GUS and in mature Mthsfa9 seeds compared to wild type seeds. (b-d) Relative transcript levels of MtHSP17.5 (b),MtHSP18.2 (c) and MtHSPr0 (d) in wild type seeds (WT), associated wild type seeds (assocWT) and two Mthsfa9 mutant alleles. Data represent the average +- S.E.M. of three technical replicates from 30 seeds. (e-h) Soluble sugar content in mature seeds of the indicated genotypes, (e) glucose, (f) sucrose, (g) stachyose, (h) verbascose. Different letters indicate significant differences between genotypes using ANOVA and post-hoc Student-Newman-Keuls comparisons $(\mathrm{P}<0.05)$. (i) Overrepresentation analysis of KEGG pathways of differentially expressed genes of mature Mthsfa9 /WT seeds.

FIGURE 3. HSFA9 is not implicated in seed longevity under dry storage but improves survival during wet storage. (a-b) Loss of germination of mature seeds from wild type (WT) associated WT and Mthsfa9 plants during storage at $75 \% \mathrm{RH}$ and $35 \mathrm{degC}$ (a) and at $100 \% \mathrm{RH}$ and $40 \mathrm{degC}$ (b). Data are the mean (+- SE) of 3 replicates of 30 seeds. (c-d) Loss of germination of mature seeds from WT (Col-0) andhsfa9 Arabidopsis thaliana plants during storage at $75 \% \mathrm{RH}$ and $35 \operatorname{degC}(\mathrm{c})$ and $100 \% \mathrm{RH}$ and $40 \mathrm{degC}$ (d). At a given time point of ageing, data are significantly different when they differ by $15 \%$ or more $\left(\chi^{2}\right.$ test, $\left.\mathrm{P}<0.05\right)$. WT, wild type, assocWT, associated WT.

FIGURE 4 . MtHSFA9 influences the depth of dormancy during late maturation. (a) Germination curves of freshly harvested seeds of wildtype, associated WT and Mthsfa9 mutants. Seeds were dried for 3d at $43 \% \mathrm{RH}$ after pod abscission before imbibition at $20^{\circ} \mathrm{C}$ in the dark. Data are the mean of three replicates of 30 seeds. (b) Germination curves of seeds of mature seeds from wildtype and Mthsfa9 mutants after stratification for $3 \mathrm{~d}$ at $4^{\circ} \mathrm{C}$. (c) Effect of after-ripening at room temperature on mean germination time of mature seeds from wildtype and $\mathrm{Mthsfa} 9$ plants grown at $20^{\circ} \mathrm{C} / 18^{\circ} \mathrm{C}$. Data are the mean of three replicates of 30 seeds. Stars indicate significant difference between WT and mutants using ANOVA and post-hoc Student-Newman-Keuls comparisons $(\mathrm{P}<0.05)$. (d) Increase in mean germination time as proxy of depth of dormancy of wildtype and hsfa9 seeds freshly harvested at the indicated time during development. 44 DAP corresponds to the point of pod abscission. Data are the mean of three replicates of 30-50 seeds. WT, wildtype, assocWT, associated WT.

FIGURE 5 . ABA and GA biosynthesis, catabolism and signaling genes are deregulated by MtHSFA9. (a) Heatmap showing significant differential transcript levels $(\log 2)$ of genes related to ABA and GA biosynthesis, reception and signaling in mature seeds of WT and two Mthsfa9 alleles. Asterisk indicates a significant difference in transcript level upon overexpression of MtHSFA9 in hairy roots. (b-d) Relative transcript levels of MtNCED4 (Medtr5g025270) (b), MtCYP707A (Medtr8g07226) (c) and MtGa2OX2 (Medtr4g096840) (d) in mature wild type seeds (WT), associated wild type seeds (assocWT) and two Mthsfa9 mutant alleles. Different letters indicate significant differences between genotypes using ANOVA and post-hoc StudentNewman-Keuls comparisons $(\mathrm{P}<0.05)$.

FIGURE 6 . ABA content and sensitivity are affected inMthsfa9 mutants. (a) ABA content in mature seeds of freshly harvested associated WT and Mthsfa9 mutants. (b) Fold reduction in the germination speed (T50) by imbibition of mature seeds of WT, assocWT and Mthsfa9 seeds by $10 \mu \mathrm{M}$ fluridone. (c) ABA sensitivity of seeds of WT, assocWT and Mthsfa9 mutants as measured by reduction of germination after incubation in different concentrations of ABA in the dark. (d) Time to $50 \%$ of germination or start of growth for intact scarified seeds or naked embryos of WT, assocWT and Mthsfa9 mutants 1 month after harvest. Seed envelopes were removed $6 \mathrm{~h}$ after imbibition. Different letters or stars indicate significant differences between genotypes using ANOVA and post-hoc Student-Newman-Keuls comparisons $(\mathrm{P}<0.05)$.

\section{Hosted file}

Figure 1.pptx available at https://authorea.com/users/321977/articles/451106-the-seed- 
specific-heat-shock-factor-a9-regulates-the-depth-of-dormancy-in-medicago-truncatulaseeds-via-aba-signaling

\section{Hosted file}

Figure 2.pptx available at https://authorea.com/users/321977/articles/451106-the-seedspecific-heat-shock-factor-a9-regulates-the-depth-of-dormancy-in-medicago-truncatulaseeds-via-aba-signaling

\section{Hosted file}

Figure 3.pptx available at https://authorea.com/users/321977/articles/451106-the-seedspecific-heat-shock-factor-a9-regulates-the-depth-of-dormancy-in-medicago-truncatulaseeds-via-aba-signaling

\section{Hosted file}

Figure 4.pptx available at https://authorea.com/users/321977/articles/451106-the-seedspecific-heat-shock-factor-a9-regulates-the-depth-of-dormancy-in-medicago-truncatulaseeds-via-aba-signaling

\section{Hosted file}

Figure 5.pptx available at https://authorea.com/users/321977/articles/451106-the-seedspecific-heat-shock-factor-a9-regulates-the-depth-of-dormancy-in-medicago-truncatulaseeds-via-aba-signaling

\section{Hosted file}

Figure 6.pptx available at https://authorea.com/users/321977/articles/451106-the-seedspecific-heat-shock-factor-a9-regulates-the-depth-of-dormancy-in-medicago-truncatulaseeds-via-aba-signaling 